Acta Crystallographica Section E

Structure Reports

Online

ISSN 1600-5368

\section{Dibromido(4,7-diazadecane-1,10-di- amine)copper(II)}

\section{Gervas E. Assey, Ray J. Butcher* and Yilma Gultneh}

Department of Chemistry, Howard University, 525 College Street NW, Washington DC 20059, USA

Correspondence e-mail: rbutcher99@yahoo.com

Received 8 August 2011; accepted 10 August 2011

Key indicators: single-crystal X-ray study; $T=110 \mathrm{~K}$; mean $\sigma(\mathrm{C}-\mathrm{C})=0.014 \AA$;

$R$ factor $=0.071 ; w R$ factor $=0.183 ;$ data-to-parameter ratio $=20.3$.

In the title compound, $\left[\mathrm{CuBr}_{2}\left(\mathrm{C}_{8} \mathrm{H}_{22} \mathrm{~N}_{4}\right)\right]$, the $\mathrm{Cu}^{\mathrm{II}}$ atom is sixcoordinate forming a distorted octahedral complex and is bonded to two axial bromide anions and four equatorial nitrogen donors. The equatorial $\mathrm{Cu}-\mathrm{N}$ bond distances range from 2.005 (8) to 2.046 (8) $\AA$ while the axial $\mathrm{Cu}-\mathrm{Br}$ distances are 2.8616 (17) and 2.9402 (17) $\AA$, thus the six-coordinate $\mathrm{Cu}$ complex shows the usual Jahn-Teller distortion. All amine hydrogen atoms participate in either inter- or intramolecular hydrogen bonding to the $\mathrm{Br}$ anions.

\section{Related literature}

For related structues, see: Lee et al. (1986). For other related literature, see: Jahn \& Teller (1937).

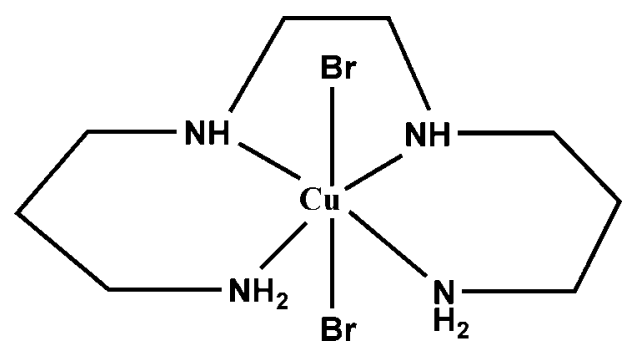

\section{Experimental}

Crystal data

$\left[\mathrm{CuBr}_{2}\left(\mathrm{C}_{8} \mathrm{H}_{22} \mathrm{~N}_{4}\right)\right]$

$M_{r}=397.66$

Orthorhombic, $P 2_{1} 2_{1} 2_{1}$ $a=6.9666(4) \AA$ $b=8.4146(6) \AA$

$c=24.0261(15) \AA$

$V=1408.45(15) \AA^{3}$

$Z=4$

Mo $K \alpha$ radiation

$\mu=7.20 \mathrm{~mm}^{-1}$

$T=110 \mathrm{~K}$

$0.47 \times 0.31 \times 0.22 \mathrm{~mm}$

Data collection

Oxford Diffraction Xcalibur diffractometer with a Ruby detector

Absorption correction: analytical (CrysAlis PRO; Oxford

Diffraction, 2007)

$T_{\text {min }}=0.157, T_{\max }=0.282$ 9561 measured reflections 2758 independent reflections 2262 reflections with $I>2 \sigma(I)$ $R_{\text {int }}=0.072$

Refinement

$R\left[F^{2}>2 \sigma\left(F^{2}\right)\right]=0.071$

$w R\left(F^{2}\right)=0.183$

$S=1.07$

2758 reflections

136 parameters

$\mathrm{H}$-atom parameters constrained

$\Delta \rho_{\max }=2.51{\mathrm{e} \AA^{-3}}^{-3}$

$\Delta \rho_{\min }=-1.98{\mathrm{e} \AA^{-3}}^{-3}$

Table 1

Hydrogen-bond geometry $\left(\AA,{ }^{\circ}\right)$.

\begin{tabular}{lllll}
\hline$D-\mathrm{H} \cdots A$ & $D-\mathrm{H}$ & $\mathrm{H} \cdots A$ & $D \cdots A$ & $D-\mathrm{H} \cdots A$ \\
\hline $\mathrm{N} 1-\mathrm{H} 1 C \cdots \mathrm{Br} 2^{\mathrm{i}}$ & 0.92 & 2.66 & $3.466(9)$ & 147 \\
$\mathrm{~N} 1-\mathrm{H} 1 D \cdots \mathrm{Br} 2$ & 0.92 & 2.80 & $3.339(8)$ & 119 \\
$\mathrm{~N} 2-\mathrm{H} 2 C \cdots \mathrm{Br} 1^{\mathrm{ii}}$ & 0.93 & 2.66 & $3.407(8)$ & 138 \\
$\mathrm{~N} 2-\mathrm{H} 2 C \cdots \mathrm{Br} 2$ & 0.93 & 3.01 & $3.519(7)$ & 116 \\
$\mathrm{~N} 3-\mathrm{H} 3 C \cdots \mathrm{Br} 1$ & 0.93 & 2.90 & $3.409(8)$ & 116 \\
$\mathrm{~N} 4-\mathrm{H} 4 C \cdots \mathrm{Br} 2^{\mathrm{i}}$ & 0.92 & 2.60 & $3.515(8)$ & 171 \\
$\mathrm{~N} 4-\mathrm{H} 4 D \cdots \mathrm{Br} 2^{\mathrm{iii}}$ & 0.92 & 2.69 & $3.425(8)$ & 138 \\
$\mathrm{~N} 4-\mathrm{H} 4 D \cdots \mathrm{Br} 1$ & 0.92 & 2.94 & $3.433(8)$ & 115
\end{tabular}

Symmetry codes: (i) $x+\frac{1}{2},-y+\frac{3}{2},-z$; (ii) $x-1, y, z$; (iii) $x+1, y, z$.

Data collection: CrysAlis PRO (Oxford Diffraction, 2007); cell refinement: CrysAlis PRO; data reduction: CrysAlis RED (Oxford Diffraction, 2007); program(s) used to solve structure: SHELXS97 (Sheldrick, 2008); program(s) used to refine structure: SHELXL97 (Sheldrick, 2008); molecular graphics: SHELXTL (Sheldrick, 2008); software used to prepare material for publication: SHELXTL.

RJB wishes to acknowledge the NSF-MRI program (grant No. CHE-0619278) for funds to purchase the diffractometer.

Supplementary data and figures for this paper are available from the IUCr electronic archives (Reference: PV2444).

\title{
References
}

Jahn, H. \& Teller, E. (1937). Proc. R. Soc. London Ser. A, pp. 220-235.

Lee, T.-Y., Lee, T.-J., Hong, C.-Y., Hsieh, M.-Y., Wu, D.-T. \& Chung, C.-S. (1986). Acta Cryst. C42, 1316-1319.

Oxford Diffraction (2007). CrysAlis PRO and CrysAlis RED. Oxford Diffraction Ltd, Abingdon, England.

Sheldrick, G. M. (2008). Acta Cryst. A64, 112-122. 


\section{supporting information}

Acta Cryst. (2011). E67, m1266 [doi:10.1107/S160053681103251X]

\section{Dibromido(4,7-diazadecane-1,10-diamine)copper(II)}

\section{Gervas E. Assey, Ray J. Butcher and Yilma Gultneh}

\section{S1. Comment}

In this study, the title compound was prepared and its structure determined by X-ray analysis. Owing to the Jahn-Teller distortion (Jahn \& Teller, 1937), the $\mathrm{Cu}(\mathrm{II})$ center adopts an axially distorted octahedral $\mathrm{CuN}_{4} \mathrm{Br}_{2}$ conformation with the axial positions are occupied by the bromide anions. The equatorial positions are occupied by the $\mathrm{N}_{4}$ set of donor nitrogen atoms and the $\mathrm{Cu} 1$ lies in the $\mathrm{N}_{4}$ plane; maximum deviation of any atom from the mean-plane formed by $\mathrm{CuN}_{4}$ fragment being 0.042 (4) for N3. The structure of a related compound containing the same linear tetramine, has been reported (Lee et al. 1986) and its structural features compared with those of other linear $\mathrm{Cu}(\mathrm{II})$ aliphatic tetraamines of the type $\mathrm{H}_{2} \mathrm{~N}\left(\mathrm{CH}_{2}\right)_{1} \mathrm{NH}-\left(\mathrm{CH}_{2}\right)_{\mathrm{m}} \mathrm{NH}\left(\mathrm{CH}_{2}\right)_{\mathrm{n}} \mathrm{NH}_{2}$ where $1, \mathrm{~m}$ and $\mathrm{n}$ are 2 or 3 . From this it can be seen that in the title complex, the equatorial $\mathrm{Cu}-\mathrm{N}$ bond distances range from 2.005 (8) to 2.046 (8) $\AA$ and are in the normal range for such bonds. However, the axial $\mathrm{Cu}-\mathrm{Br}$ distances are elongated at 2.8616 (17) and 2.9402 (17) $\AA$, thus the 6-coordinate $\mathrm{Cu}$ complex shows the usual Jahn-Teller distortion. All amine H's participate in either inter or intramolecular hydrogen bonding to the Br anions.

\section{S2. Experimental}

The title compound was obtained as a byproduct of an attempt to prepare copper complexes of ethylenediamine $\mathrm{N}, \mathrm{N}$-bis(propylsalicylaldimine). A solution of N, $N$-bis(3-aminopropylethylene)diamine (5 g, $30.52 \mathrm{mmol})$ in methanol (20 ml) was added dropwise to a solution of salicylaldehyde $(7.45 \mathrm{~g}, 61.04 \mathrm{mmol})$ in methanol $(20 \mathrm{ml})$. The mixture was refluxed overnight while stirring with magnetic stirrer. Then the reaction mixture was evaporated under reduced pressure. An oily orange product was obtained which later solidified into a yellow compound, [2-(3-amino-propylamino)-ethyl]propane-1,3-diamine-bis(salicyladimine), used as a ligand $\left(\mathrm{H}_{2} L_{4}\right)$ in the subsequent reaction. The synthesis of the title complex was achieved by the reaction of $\mathrm{CuBr}(1.5 \mathrm{~g}, 10.5 \mathrm{mmol})$ in methanol $(20 \mathrm{ml})$ with of the ligand $\mathrm{H}_{2} L_{4}(2 \mathrm{~g}, 5.2$ mmol) dissolved in $\mathrm{CH}_{2} \mathrm{Cl}_{2}(25 \mathrm{ml})$. The ligand solution was added drop-wise to the solution of the metal salt and stirred at room temperature for $24 \mathrm{~h}$. The mixture was then concentrated by evaporation under reduced pressure to afford a thick greenish liquid. Part of the complex was dissolved in dimethyl formamide (DMF), filtered and layered with diethyl ether for slow diffusion and X-ray quality crystals were obtained.

\section{S3. Refinement}

$\mathrm{H}$ atoms were placed in geometrically idealized positions and constrained to ride on their parent atoms with a $\mathrm{C}-\mathrm{H}$ distance of $0.99 \AA$ and $\mathrm{N}-\mathrm{H}$ distances of 0.92 (primary amine) and 0.93 (secondary amine) with $U_{\text {iso }}(\mathrm{H})=1.2 U_{\text {eq }}(\mathrm{C}, \mathrm{N})$. Even though a face-indexed absorption correction was carried out, the thermal parameters for $\mathrm{C} 3, \mathrm{C} 6, \mathrm{C} 7$, and $\mathrm{N} 4$ atoms did not behave well and thus were restrained using ISOR command in SHELXL. The crystal was originally refined as a racemic twin with components 0.87 (3):0.13 (3). However, as the absolute configuration was not established unambiguously, the data were merged. In addition, the highest peak $\left(2.50 \mathrm{e}^{-} / \AA^{3}, 0.70 \AA\right.$ from $\left.\mathrm{Cu}\right)$ and deepest hole $(-1.98$ 
$\mathrm{e}^{-} / \AA^{3}, 0.54 \AA$ from $\left.\mathrm{Br} 2\right)$ are indicative of the problems with both the racemic twinning and absorption effects.

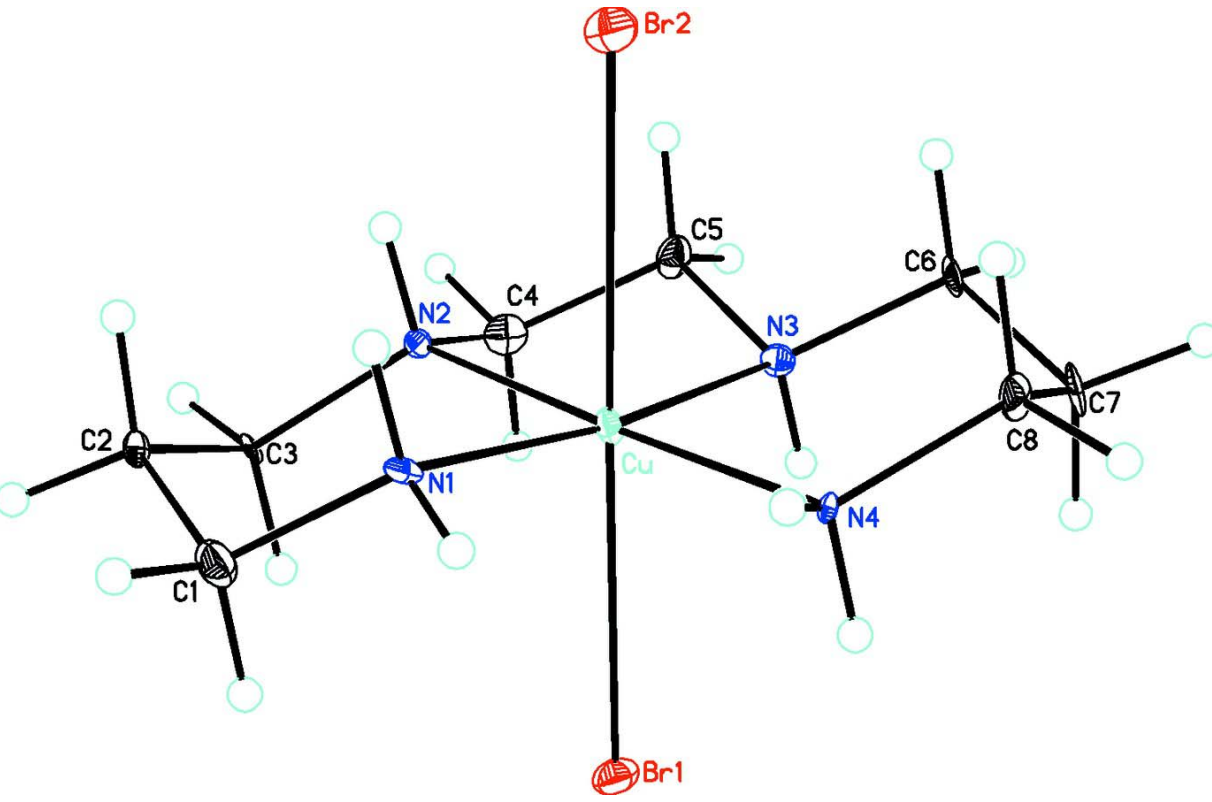

\section{Figure 1}

An ORTEP drawing of the title complex showing atom labeling. Thermal ellipsoids are drawn at the $30 \%$ probability level. 


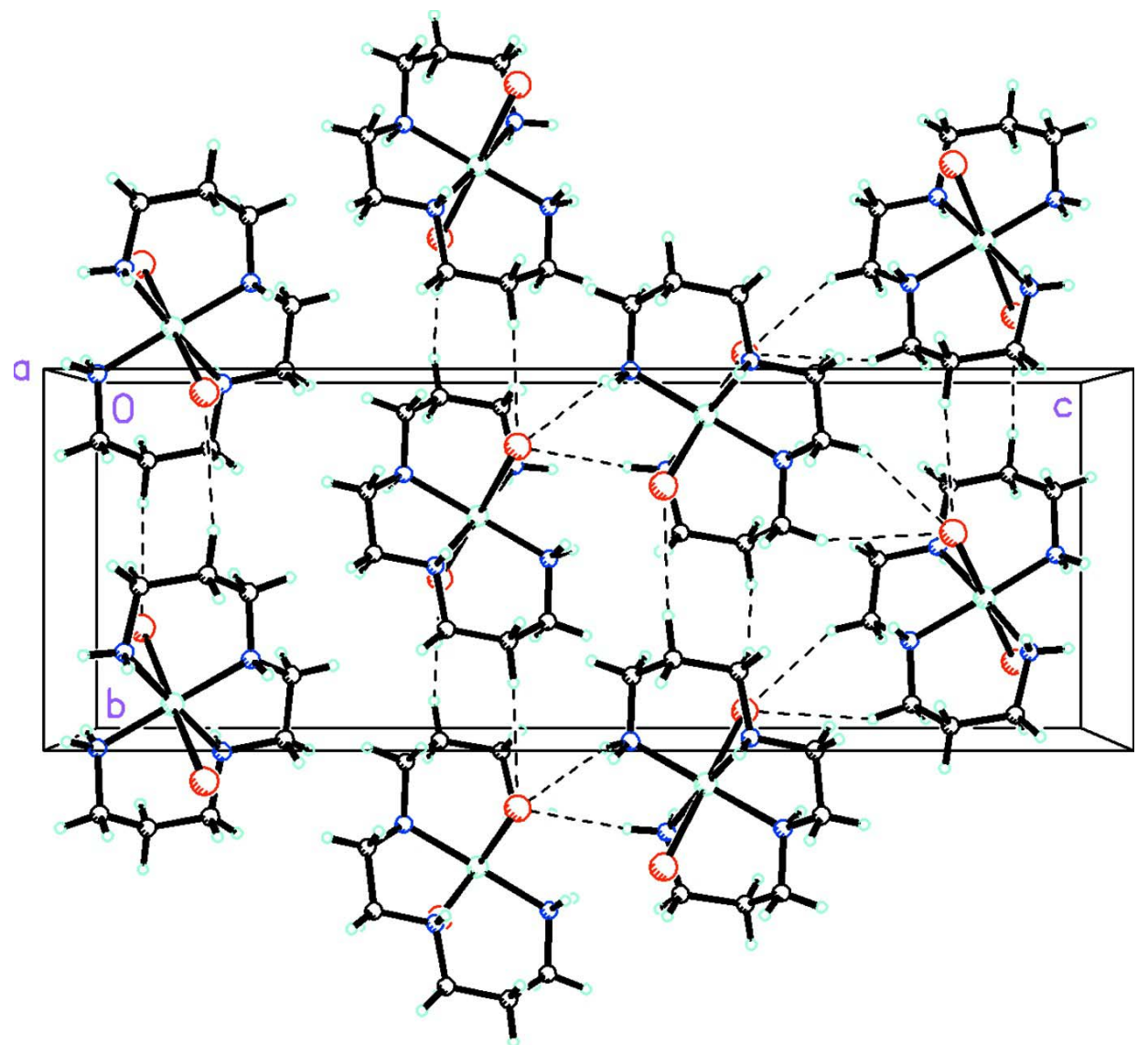

Figure 2

The molecular packing for the title compound viewed down the $a$ axis. Hydrogen bonds are showed by dashed lines.

\section{Dibromido(4,7-diazadecane-1,10-diamine)copper(II)}

\section{Crystal data}

$\left[\mathrm{CuBr}_{2}\left(\mathrm{C}_{8} \mathrm{H}_{22} \mathrm{~N}_{4}\right)\right]$

$M_{r}=397.66$

Orthorhombic, $P 2_{1} 2_{1} 2_{1}$

Hall symbol: P $2 \mathrm{ac} 2 \mathrm{ab}$

$a=6.9666(4) \AA$

$b=8.4146(6) \AA$

$c=24.0261(15) \AA$

$V=1408.45(15) \AA^{3}$

$Z=4$

\section{Data collection}

Goniometer Xcalibur, detector Ruby (Gemini Mo)

diffractometer

Radiation source: Enhance (Mo) X-ray Source

Graphite monochromator

Detector resolution: 10.5081 pixels $\mathrm{mm}^{-1}$

$\omega$ scans

Absorption correction: analytical

(CrysAlis PRO; Oxford Diffraction, 2007)
$F(000)=788$

$D_{\mathrm{x}}=1.875 \mathrm{Mg} \mathrm{m}^{-3}$

Mo $K \alpha$ radiation, $\lambda=0.71073 \AA$

Cell parameters from 4805 reflections

$\theta=4.6-32.8^{\circ}$

$\mu=7.20 \mathrm{~mm}^{-1}$

$T=110 \mathrm{~K}$

Prism, dark blue

$0.47 \times 0.31 \times 0.22 \mathrm{~mm}$

$T_{\min }=0.157, T_{\max }=0.282$

9561 measured reflections

2758 independent reflections

2262 reflections with $I>2 \sigma(I)$

$R_{\text {int }}=0.072$

$\theta_{\text {max }}=32.8^{\circ}, \theta_{\text {min }}=4.6^{\circ}$

$h=-10 \rightarrow 9$

$k=-12 \rightarrow 11$

$l=-36 \rightarrow 35$ 


\section{Refinement}

Refinement on $F^{2}$

Least-squares matrix: full

$R\left[F^{2}>2 \sigma\left(F^{2}\right)\right]=0.071$

$w R\left(F^{2}\right)=0.183$

$S=1.07$

2758 reflections

136 parameters

24 restraints

Primary atom site location: structure-invariant direct methods
Secondary atom site location: difference Fourier map

Hydrogen site location: inferred from neighbouring sites

$\mathrm{H}$-atom parameters constrained

$w=1 /\left[\sigma^{2}\left(F_{\mathrm{o}}{ }^{2}\right)+(0.103 P)^{2}+8.8289 P\right]$ where $P=\left(F_{\mathrm{o}}{ }^{2}+2 F_{\mathrm{c}}{ }^{2}\right) / 3$

$(\Delta / \sigma)_{\max }<0.001$

$\Delta \rho_{\max }=2.51 \mathrm{e} \AA^{-3}$

$\Delta \rho_{\min }=-1.98$ e $\AA^{-3}$

Special details

Geometry. All s.u.'s (except the s.u. in the dihedral angle between two l.s. planes) are estimated using the full covariance matrix. The cell s.u.'s are taken into account individually in the estimation of s.u.'s in distances, angles and torsion angles; correlations between s.u.'s in cell parameters are only used when they are defined by crystal symmetry. An approximate (isotropic) treatment of cell s.u.'s is used for estimating s.u.'s involving l.s. planes.

Refinement. Refinement of $F^{2}$ against ALL reflections. The weighted $R$-factor $w R$ and goodness of fit $S$ are based on $F^{2}$, conventional $R$-factors $R$ are based on $F$, with $F$ set to zero for negative $F^{2}$. The threshold expression of $F^{2}>2 \sigma\left(F^{2}\right)$ is used only for calculating $R$-factors(gt) $e t c$. and is not relevant to the choice of reflections for refinement. $R$-factors based on $F^{2}$ are statistically about twice as large as those based on $F$, and $R$ - factors based on ALL data will be even larger.

Fractional atomic coordinates and isotropic or equivalent isotropic displacement parameters $\left(\AA^{2}\right)$

\begin{tabular}{lllll}
\hline & $x$ & $y$ & $z$ & $U_{\text {iso }} / U_{\mathrm{eq}}$ \\
\hline $\mathrm{Cu}$ & $0.84387(19)$ & $0.88632(13)$ & $0.11140(4)$ & $0.0099(2)$ \\
$\mathrm{Br} 1$ & $1.15943(15)$ & $1.06828(12)$ & $0.15326(4)$ & $0.0187(2)$ \\
$\mathrm{Br} 2$ & $0.52369(16)$ & $0.69368(14)$ & $0.06892(5)$ & $0.0225(3)$ \\
$\mathrm{N} 1$ & $0.8009(12)$ & $1.0062(10)$ & $0.0394(3)$ & $0.0132(16)$ \\
$\mathrm{H} 1 \mathrm{C}$ & 0.9013 & 0.9823 & 0.0160 & $0.016^{*}$ \\
$\mathrm{H} 1 \mathrm{D}$ & 0.6914 & 0.9661 & 0.0233 & $0.016^{*}$ \\
$\mathrm{~N} 2$ & $0.6456(12)$ & $1.0252(8)$ & $0.1509(3)$ & $0.0106(13)$ \\
$\mathrm{H} 2 \mathrm{C}$ & 0.5256 & 0.9901 & 0.1392 & $0.013^{*}$ \\
$\mathrm{~N} 3$ & $0.8538(13)$ & $0.7653(9)$ & $0.1852(3)$ & $0.0125(14)$ \\
$\mathrm{H} 3 \mathrm{C}$ & 0.9601 & 0.8035 & 0.2044 & $0.015^{*}$ \\
$\mathrm{~N} 4$ & $1.0360(12)$ & $0.7415(9)$ & $0.0756(3)$ & $0.0110(14)$ \\
$\mathrm{H} 4 \mathrm{C}$ & 1.0215 & 0.7508 & 0.0376 & $0.013^{*}$ \\
$\mathrm{H} 4 \mathrm{D}$ & 1.1562 & 0.7796 & 0.0841 & $0.013^{*}$ \\
$\mathrm{C} 1$ & $0.7815(17)$ & $1.1841(12)$ & $0.0409(4)$ & $0.0178(19)$ \\
$\mathrm{H} 1 \mathrm{~A}$ & 0.9031 & 1.2316 & 0.0542 & $0.021^{*}$ \\
$\mathrm{H} 1 \mathrm{~B}$ & 0.7571 & 1.2240 & 0.0028 & $0.021^{*}$ \\
$\mathrm{C} 2$ & $0.6201(14)$ & $1.2343(11)$ & $0.0785(4)$ & $0.0143(18)$ \\
$\mathrm{H} 2 \mathrm{~A}$ & 0.5018 & 1.1786 & 0.0666 & $0.017^{*}$ \\
$\mathrm{H} 2 \mathrm{~B}$ & 0.5983 & 1.3496 & 0.0734 & $0.017^{*}$ \\
$\mathrm{C} 3$ & $0.6518(15)$ & $1.2020(11)$ & $0.1399(4)$ & $0.0123(15)$ \\
$\mathrm{H} 3 \mathrm{~A}$ & 0.7780 & 1.2451 & 0.1514 & $0.015^{*}$ \\
$\mathrm{H} 3 \mathrm{~B}$ & 0.5511 & 1.2559 & 0.1620 & $0.015^{*}$ \\
$\mathrm{C} 4$ & $0.6583(17)$ & $0.9909(12)$ & $0.2110(4)$ & $0.0160(17)$ \\
$\mathrm{H} 4 \mathrm{~A}$ & 0.5407 & 1.0279 & 0.2302 & $0.019^{*}$ \\
$\mathrm{H} 4 \mathrm{~B}$ & 0.7700 & 1.0467 & 0.2274 & $0.019^{*}$ \\
& & & &
\end{tabular}




$\begin{array}{lllll}\text { C5 } & 0.6801(16) & 0.8160(13) & 0.2179(4) & 0.0179(19) \\ \text { H5A } & 0.5644 & 0.7606 & 0.2039 & 0.021^{*} \\ \text { H5B } & 0.6970 & 0.7891 & 0.2577 & 0.021^{*} \\ \text { C6 } & 0.8734(14) & 0.5888(11) & 0.1836(4) & 0.0138(18) \\ \text { H6A } & 0.7584 & 0.5421 & 0.1658 & 0.017^{*} \\ \text { H6B } & 0.8813 & 0.5473 & 0.2221 & 0.017^{*} \\ \text { C7 } & 1.0503(15) & 0.5394(11) & 0.1517(4) & 0.0158(18) \\ \text { H7A } & 1.0739 & 0.4248 & 0.1581 & 0.019^{*} \\ \text { H7B } & 1.1623 & 0.5986 & 0.1663 & 0.019^{*} \\ \text { C8 } & 1.0339(15) & 0.5691(11) & 0.0892(4) & 0.0142(17) \\ \text { H8A } & 1.1419 & 0.5161 & 0.0700 & 0.017^{*} \\ \text { H8B } & 0.9130 & 0.5216 & 0.0754 & 0.017^{*} \\ \end{array}$

Atomic displacement parameters $\left(\AA^{2}\right)$

\begin{tabular}{lllllll}
\hline & $U^{11}$ & $U^{22}$ & $U^{33}$ & $U^{12}$ & $U^{13}$ & $U^{23}$ \\
\hline $\mathrm{Cu}$ & $0.0119(5)$ & $0.0064(4)$ & $0.0113(4)$ & $0.0022(4)$ & $0.0026(4)$ & $0.0007(4)$ \\
$\mathrm{Br} 1$ & $0.0107(4)$ & $0.0185(5)$ & $0.0270(5)$ & $-0.0016(4)$ & $-0.0007(4)$ & $-0.0062(4)$ \\
$\mathrm{Br} 2$ & $0.0169(5)$ & $0.0213(5)$ & $0.0293(5)$ & $-0.0013(4)$ & $-0.0023(4)$ & $-0.0026(4)$ \\
$\mathrm{N} 1$ & $0.011(4)$ & $0.011(4)$ & $0.017(4)$ & $-0.004(3)$ & $-0.001(3)$ & $0.002(3)$ \\
$\mathrm{N} 2$ & $0.010(3)$ & $0.005(3)$ & $0.017(3)$ & $0.001(3)$ & $-0.001(3)$ & $-0.001(3)$ \\
$\mathrm{N} 3$ & $0.011(3)$ & $0.012(3)$ & $0.015(3)$ & $-0.001(3)$ & $0.003(3)$ & $0.002(3)$ \\
$\mathrm{N} 4$ & $0.008(3)$ & $0.009(3)$ & $0.016(3)$ & $0.002(2)$ & $0.002(2)$ & $0.000(2)$ \\
$\mathrm{C} 1$ & $0.023(5)$ & $0.012(4)$ & $0.018(4)$ & $-0.002(4)$ & $0.003(4)$ & $0.005(4)$ \\
$\mathrm{C} 2$ & $0.012(4)$ & $0.005(3)$ & $0.026(5)$ & $0.004(3)$ & $0.001(3)$ & $0.002(3)$ \\
$\mathrm{C} 3$ & $0.012(3)$ & $0.006(3)$ & $0.019(3)$ & $0.001(3)$ & $0.001(3)$ & $0.002(3)$ \\
$\mathrm{C} 4$ & $0.017(4)$ & $0.021(4)$ & $0.010(3)$ & $0.002(4)$ & $0.004(4)$ & $-0.002(3)$ \\
$\mathrm{C} 5$ & $0.019(5)$ & $0.020(4)$ & $0.015(4)$ & $0.006(4)$ & $0.006(4)$ & $0.003(4)$ \\
$\mathrm{C} 6$ & $0.016(4)$ & $0.009(3)$ & $0.017(3)$ & $0.003(3)$ & $0.002(3)$ & $0.004(3)$ \\
$\mathrm{C} 7$ & $0.020(4)$ & $0.009(3)$ & $0.018(3)$ & $0.005(3)$ & $0.001(3)$ & $0.003(3)$ \\
$\mathrm{C} 8$ & $0.016(4)$ & $0.009(4)$ & $0.017(4)$ & $0.004(4)$ & $0.004(3)$ & $-0.003(3)$ \\
& & & & &
\end{tabular}

Geometric parameters $\left(\hat{A},{ }^{\circ}\right)$

\begin{tabular}{llll}
\hline $\mathrm{Cu}-\mathrm{N} 4$ & $2.005(8)$ & $\mathrm{C} 1-\mathrm{H} 1 \mathrm{~B}$ & 0.9900 \\
$\mathrm{Cu}-\mathrm{N} 1$ & $2.025(8)$ & $\mathrm{C} 2-\mathrm{C} 3$ & $1.517(13)$ \\
$\mathrm{Cu}-\mathrm{N} 2$ & $2.043(8)$ & $\mathrm{C} 2-\mathrm{H} 2 \mathrm{~A}$ & 0.9900 \\
$\mathrm{Cu}-\mathrm{N} 3$ & $2.046(8)$ & $\mathrm{C} 2-\mathrm{H} 2 \mathrm{~B}$ & 0.9900 \\
$\mathrm{Cu}-\mathrm{Br} 1$ & $2.8616(17)$ & $\mathrm{C} 3-\mathrm{H} 3 \mathrm{~A}$ & 0.9900 \\
$\mathrm{Cu}-\mathrm{Br} 2$ & $2.9402(17)$ & $\mathrm{C} 3-\mathrm{H} 3 \mathrm{~B}$ & 0.9900 \\
$\mathrm{~N} 1-\mathrm{C} 1$ & $1.503(13)$ & $\mathrm{C} 4-\mathrm{C} 5$ & $1.489(15)$ \\
$\mathrm{N} 1-\mathrm{H} 1 \mathrm{C}$ & 0.9200 & $\mathrm{C} 4-\mathrm{H} 4 \mathrm{~A}$ & 0.9900 \\
$\mathrm{~N} 1-\mathrm{H} 1 \mathrm{D}$ & 0.9200 & $\mathrm{C} 4-\mathrm{H} 4 \mathrm{~B}$ & 0.9900 \\
$\mathrm{~N} 2-\mathrm{C} 4$ & $1.476(12)$ & $\mathrm{C} 5-\mathrm{H} 5 \mathrm{~A}$ & 0.9900 \\
$\mathrm{~N} 2-\mathrm{C} 3$ & $1.512(11)$ & $\mathrm{C} 5-\mathrm{H} 5 \mathrm{~B}$ & 0.9900 \\
$\mathrm{~N} 2-\mathrm{H} 2 \mathrm{C}$ & 0.9300 & $\mathrm{C} 6-\mathrm{C} 7$ & $1.510(14)$ \\
$\mathrm{N} 3-\mathrm{C} 6$ & $1.492(11)$ & $\mathrm{C} 6-\mathrm{H} 6 \mathrm{~A}$ & 0.9900 \\
$\mathrm{~N} 3-\mathrm{C} 5$ & $1.504(13)$ & $\mathrm{C} 6-\mathrm{H} 6 \mathrm{~B}$ & 0.9900
\end{tabular}




\begin{tabular}{|c|c|c|c|}
\hline $\mathrm{N} 3-\mathrm{H} 3 \mathrm{C}$ & 0.9300 & $\mathrm{C} 7-\mathrm{C} 8$ & $1.526(14)$ \\
\hline $\mathrm{N} 4-\mathrm{C} 8$ & $1.487(12)$ & C7-H7A & 0.9900 \\
\hline $\mathrm{N} 4-\mathrm{H} 4 \mathrm{C}$ & 0.9200 & C7-H7B & 0.9900 \\
\hline N4-H4D & 0.9200 & $\mathrm{C} 8-\mathrm{H} 8 \mathrm{~A}$ & 0.9900 \\
\hline $\mathrm{C} 1-\mathrm{C} 2$ & 1.503 (14) & $\mathrm{C} 8-\mathrm{H} 8 \mathrm{~B}$ & 0.9900 \\
\hline $\mathrm{C} 1-\mathrm{H} 1 \mathrm{~A}$ & 0.9900 & & \\
\hline $\mathrm{N} 4-\mathrm{Cu}-\mathrm{N} 1$ & $92.0(3)$ & $\mathrm{H} 1 \mathrm{~A}-\mathrm{C} 1-\mathrm{H} 1 \mathrm{~B}$ & 108.0 \\
\hline $\mathrm{N} 4-\mathrm{Cu}-\mathrm{N} 2$ & $177.1(3)$ & $\mathrm{C} 1-\mathrm{C} 2-\mathrm{C} 3$ & $115.2(8)$ \\
\hline $\mathrm{N} 1-\mathrm{Cu}-\mathrm{N} 2$ & $90.7(3)$ & $\mathrm{C} 1-\mathrm{C} 2-\mathrm{H} 2 \mathrm{~A}$ & 108.5 \\
\hline $\mathrm{N} 4-\mathrm{Cu}-\mathrm{N} 3$ & 92.7 (3) & $\mathrm{C} 3-\mathrm{C} 2-\mathrm{H} 2 \mathrm{~A}$ & 108.5 \\
\hline $\mathrm{N} 1-\mathrm{Cu}-\mathrm{N} 3$ & $173.4(4)$ & $\mathrm{C} 1-\mathrm{C} 2-\mathrm{H} 2 \mathrm{~B}$ & 108.5 \\
\hline $\mathrm{N} 2-\mathrm{Cu}-\mathrm{N} 3$ & $84.6(3)$ & $\mathrm{C} 3-\mathrm{C} 2-\mathrm{H} 2 \mathrm{~B}$ & 108.5 \\
\hline $\mathrm{N} 4-\mathrm{Cu}-\mathrm{Br} 1$ & $87.9(2)$ & $\mathrm{H} 2 \mathrm{~A}-\mathrm{C} 2-\mathrm{H} 2 \mathrm{~B}$ & 107.5 \\
\hline $\mathrm{N} 1-\mathrm{Cu}-\mathrm{Br} 1$ & $98.5(2)$ & $\mathrm{N} 2-\mathrm{C} 3-\mathrm{C} 2$ & $110.0(7)$ \\
\hline $\mathrm{N} 2-\mathrm{Cu}-\mathrm{Br} 1$ & $92.9(2)$ & $\mathrm{N} 2-\mathrm{C} 3-\mathrm{H} 3 \mathrm{~A}$ & 109.7 \\
\hline $\mathrm{N} 3-\mathrm{Cu}-\mathrm{Br} 1$ & $86.3(3)$ & $\mathrm{C} 2-\mathrm{C} 3-\mathrm{H} 3 \mathrm{~A}$ & 109.7 \\
\hline $\mathrm{N} 4-\mathrm{Cu}-\mathrm{Br} 2$ & $91.3(2)$ & $\mathrm{N} 2-\mathrm{C} 3-\mathrm{H} 3 \mathrm{~B}$ & 109.7 \\
\hline $\mathrm{N} 1-\mathrm{Cu}-\mathrm{Br} 2$ & $82.3(2)$ & $\mathrm{C} 2-\mathrm{C} 3-\mathrm{H} 3 \mathrm{~B}$ & 109.7 \\
\hline $\mathrm{N} 2-\mathrm{Cu}-\mathrm{Br} 2$ & $87.9(2)$ & $\mathrm{H} 3 \mathrm{~A}-\mathrm{C} 3-\mathrm{H} 3 \mathrm{~B}$ & 108.2 \\
\hline $\mathrm{N} 3-\mathrm{Cu}-\mathrm{Br} 2$ & $93.0(3)$ & $\mathrm{N} 2-\mathrm{C} 4-\mathrm{C} 5$ & $107.9(8)$ \\
\hline $\mathrm{Br} 1-\mathrm{Cu}-\mathrm{Br} 2$ & $178.89(6)$ & $\mathrm{N} 2-\mathrm{C} 4-\mathrm{H} 4 \mathrm{~A}$ & 110.1 \\
\hline $\mathrm{C} 1-\mathrm{N} 1-\mathrm{Cu}$ & $119.3(7)$ & $\mathrm{C} 5-\mathrm{C} 4-\mathrm{H} 4 \mathrm{~A}$ & 110.1 \\
\hline $\mathrm{C} 1-\mathrm{N} 1-\mathrm{H} 1 \mathrm{C}$ & 107.5 & $\mathrm{~N} 2-\mathrm{C} 4-\mathrm{H} 4 \mathrm{~B}$ & 110.1 \\
\hline $\mathrm{Cu}-\mathrm{N} 1-\mathrm{H} 1 \mathrm{C}$ & 107.5 & $\mathrm{C} 5-\mathrm{C} 4-\mathrm{H} 4 \mathrm{~B}$ & 110.1 \\
\hline $\mathrm{C} 1-\mathrm{N} 1-\mathrm{H} 1 \mathrm{D}$ & 107.5 & $\mathrm{H} 4 \mathrm{~A}-\mathrm{C} 4-\mathrm{H} 4 \mathrm{~B}$ & 108.4 \\
\hline $\mathrm{Cu}-\mathrm{N} 1-\mathrm{H} 1 \mathrm{D}$ & 107.5 & $\mathrm{C} 4-\mathrm{C} 5-\mathrm{N} 3$ & $107.7(9)$ \\
\hline $\mathrm{H} 1 \mathrm{C}-\mathrm{N} 1-\mathrm{H} 1 \mathrm{D}$ & 107.0 & $\mathrm{C} 4-\mathrm{C} 5-\mathrm{H} 5 \mathrm{~A}$ & 110.2 \\
\hline $\mathrm{C} 4-\mathrm{N} 2-\mathrm{C} 3$ & $111.2(7)$ & $\mathrm{N} 3-\mathrm{C} 5-\mathrm{H} 5 \mathrm{~A}$ & 110.2 \\
\hline $\mathrm{C} 4-\mathrm{N} 2-\mathrm{Cu}$ & $107.6(6)$ & $\mathrm{C} 4-\mathrm{C} 5-\mathrm{H} 5 \mathrm{~B}$ & 110.2 \\
\hline $\mathrm{C} 3-\mathrm{N} 2-\mathrm{Cu}$ & $117.6(6)$ & $\mathrm{N} 3-\mathrm{C} 5-\mathrm{H} 5 \mathrm{~B}$ & 110.2 \\
\hline $\mathrm{C} 4-\mathrm{N} 2-\mathrm{H} 2 \mathrm{C}$ & 106.6 & $\mathrm{H} 5 \mathrm{~A}-\mathrm{C} 5-\mathrm{H} 5 \mathrm{~B}$ & 108.5 \\
\hline $\mathrm{C} 3-\mathrm{N} 2-\mathrm{H} 2 \mathrm{C}$ & 106.6 & $\mathrm{~N} 3-\mathrm{C} 6-\mathrm{C} 7$ & $111.2(8)$ \\
\hline $\mathrm{Cu}-\mathrm{N} 2-\mathrm{H} 2 \mathrm{C}$ & 106.6 & $\mathrm{~N} 3-\mathrm{C} 6-\mathrm{H} 6 \mathrm{~A}$ & 109.4 \\
\hline $\mathrm{C} 6-\mathrm{N} 3-\mathrm{C} 5$ & $111.7(8)$ & $\mathrm{C} 7-\mathrm{C} 6-\mathrm{H} 6 \mathrm{~A}$ & 109.4 \\
\hline $\mathrm{C} 6-\mathrm{N} 3-\mathrm{Cu}$ & $118.5(6)$ & $\mathrm{N} 3-\mathrm{C} 6-\mathrm{H} 6 \mathrm{~B}$ & 109.4 \\
\hline $\mathrm{C} 5-\mathrm{N} 3-\mathrm{Cu}$ & $106.5(6)$ & $\mathrm{C} 7-\mathrm{C} 6-\mathrm{H} 6 \mathrm{~B}$ & 109.4 \\
\hline $\mathrm{C} 6-\mathrm{N} 3-\mathrm{H} 3 \mathrm{C}$ & 106.5 & $\mathrm{H} 6 \mathrm{~A}-\mathrm{C} 6-\mathrm{H} 6 \mathrm{~B}$ & 108.0 \\
\hline $\mathrm{C} 5-\mathrm{N} 3-\mathrm{H} 3 \mathrm{C}$ & 106.5 & $\mathrm{C} 6-\mathrm{C} 7-\mathrm{C} 8$ & $113.1(8)$ \\
\hline $\mathrm{Cu}-\mathrm{N} 3-\mathrm{H} 3 \mathrm{C}$ & 106.5 & $\mathrm{C} 6-\mathrm{C} 7-\mathrm{H} 7 \mathrm{~A}$ & 109.0 \\
\hline $\mathrm{C} 8-\mathrm{N} 4-\mathrm{Cu}$ & $119.4(6)$ & $\mathrm{C} 8-\mathrm{C} 7-\mathrm{H} 7 \mathrm{~A}$ & 109.0 \\
\hline $\mathrm{C} 8-\mathrm{N} 4-\mathrm{H} 4 \mathrm{C}$ & 107.5 & $\mathrm{C} 6-\mathrm{C} 7-\mathrm{H} 7 \mathrm{~B}$ & 109.0 \\
\hline $\mathrm{Cu}-\mathrm{N} 4-\mathrm{H} 4 \mathrm{C}$ & 107.5 & $\mathrm{C} 8-\mathrm{C} 7-\mathrm{H} 7 \mathrm{~B}$ & 109.0 \\
\hline $\mathrm{C} 8-\mathrm{N} 4-\mathrm{H} 4 \mathrm{D}$ & 107.5 & $\mathrm{H} 7 \mathrm{~A}-\mathrm{C} 7-\mathrm{H} 7 \mathrm{~B}$ & 107.8 \\
\hline $\mathrm{Cu}-\mathrm{N} 4-\mathrm{H} 4 \mathrm{D}$ & 107.5 & $\mathrm{~N} 4-\mathrm{C} 8-\mathrm{C} 7$ & $112.1(8)$ \\
\hline $\mathrm{H} 4 \mathrm{C}-\mathrm{N} 4-\mathrm{H} 4 \mathrm{D}$ & 107.0 & $\mathrm{~N} 4-\mathrm{C} 8-\mathrm{H} 8 \mathrm{~A}$ & 109.2 \\
\hline $\mathrm{C} 2-\mathrm{C} 1-\mathrm{N} 1$ & $111.2(8)$ & $\mathrm{C} 7-\mathrm{C} 8-\mathrm{H} 8 \mathrm{~A}$ & 109.2 \\
\hline $\mathrm{C} 2-\mathrm{C} 1-\mathrm{H} 1 \mathrm{~A}$ & 109.4 & $\mathrm{~N} 4-\mathrm{C} 8-\mathrm{H} 8 \mathrm{~B}$ & 109.2 \\
\hline
\end{tabular}




$\begin{array}{llll}\mathrm{N} 1-\mathrm{C} 1-\mathrm{H} 1 \mathrm{~A} & 109.4 & \mathrm{C} 7-\mathrm{C} 8-\mathrm{H} 8 \mathrm{~B} & 109.2 \\ \mathrm{C} 2-\mathrm{C} 1-\mathrm{H} 1 \mathrm{~B} & 109.4 & \mathrm{H} 8 \mathrm{~A}-\mathrm{C} 8-\mathrm{H} 8 \mathrm{~B} & 107.9 \\ \mathrm{~N} 1-\mathrm{C} 1-\mathrm{H} 1 \mathrm{~B} & 109.4 & & \\ & & \mathrm{~N} 1-\mathrm{Cu}-\mathrm{N} 4-\mathrm{C} 8 & -138.9(7) \\ \mathrm{N} 4-\mathrm{Cu}-\mathrm{N} 1-\mathrm{C} 1 & -139.1(8) & \mathrm{N} 3-\mathrm{Cu}-\mathrm{N} 4-\mathrm{C} 8 & 36.5(7) \\ \mathrm{N} 2-\mathrm{Cu}-\mathrm{N} 1-\mathrm{C} 1 & 42.1(8) & \mathrm{B} 1-\mathrm{Cu}-\mathrm{N} 4-\mathrm{C} 8 & 122.7(7) \\ \mathrm{Br} 1-\mathrm{Cu}-\mathrm{N} 1-\mathrm{C} 1 & -50.9(8) & \mathrm{B} 2-\mathrm{Cu}-\mathrm{N} 4-\mathrm{C} 8 & -56.6(7) \\ \mathrm{Br} 2-\mathrm{Cu}-\mathrm{N} 1-\mathrm{C} 1 & 129.9(8) & \mathrm{N} 1-\mathrm{C} 1-\mathrm{C} 1-\mathrm{C} 2 & -\mathrm{C} 3 \\ \mathrm{~N} 1-\mathrm{Cu}-\mathrm{N} 2-\mathrm{C} 4 & -170.1(6) & \mathrm{C} 4-\mathrm{N} 2-\mathrm{C} 3-\mathrm{C} 2 & 67.5(11) \\ \mathrm{N} 3-\mathrm{Cu}-\mathrm{N} 2-\mathrm{C} 4 & 14.4(6) & \mathrm{Cu}-\mathrm{N} 2-\mathrm{C} 3-\mathrm{C} 2 & -174.8(8) \\ \mathrm{Br} 1-\mathrm{Cu}-\mathrm{N} 2-\mathrm{C} 4 & -71.6(6) & \mathrm{C} 1-\mathrm{C} 2-\mathrm{C} 3-\mathrm{N} 2 & 60.6(10) \\ \mathrm{Br} 2-\mathrm{Cu}-\mathrm{N} 2-\mathrm{C} 4 & 107.6(6) & \mathrm{C} 3-\mathrm{N} 2-\mathrm{C} 4-\mathrm{C} 5 & -69.7(11) \\ \mathrm{N} 1-\mathrm{Cu}-\mathrm{N} 2-\mathrm{C} 3 & -43.7(7) & \mathrm{Cu}-\mathrm{N} 2-\mathrm{C} 4-\mathrm{C} 5 & -171.9(9) \\ \mathrm{N} 3-\mathrm{Cu}-\mathrm{N} 2-\mathrm{C} 3 & 140.8(7) & \mathrm{N} 2-\mathrm{C} 4-\mathrm{C} 5-\mathrm{N} 3 & -41.8(10) \\ \mathrm{Br} 1-\mathrm{Cu}-\mathrm{N} 2-\mathrm{C} 3 & 54.8(6) & \mathrm{C} 6-\mathrm{N} 3-\mathrm{C} 5-\mathrm{C} 4 & 56.4(11) \\ \mathrm{Br} 2-\mathrm{Cu}-\mathrm{N} 2-\mathrm{C} 3 & -126.0(6) & \mathrm{Cu}-\mathrm{N} 3-\mathrm{C} 5-\mathrm{C} 4 & -172.8(8) \\ \mathrm{N} 4-\mathrm{Cu}-\mathrm{N} 3-\mathrm{C} 6 & -37.2(8) & \mathrm{C} 5-\mathrm{N} 3-\mathrm{C} 6-\mathrm{C} 7 & -42.0(9) \\ \mathrm{N} 2-\mathrm{Cu}-\mathrm{N} 3-\mathrm{C} 6 & 141.9(8) & \mathrm{Cu}-\mathrm{N} 3-\mathrm{C} 6-\mathrm{C} 7 & -178.8(8) \\ \mathrm{Br} 1-\mathrm{Cu}-\mathrm{N} 3-\mathrm{C} 6 & -124.9(7) & \mathrm{N} 3-\mathrm{C} 6-\mathrm{C} 7-\mathrm{C} 8 & 56.9(10) \\ \mathrm{Br} 2-\mathrm{Cu}-\mathrm{N} 3-\mathrm{C} 6 & 54.3(7) & \mathrm{Cu}-\mathrm{N} 4-\mathrm{C} 8-\mathrm{C} 7 & -70.4(11) \\ \mathrm{N} 4-\mathrm{Cu}-\mathrm{N} 3-\mathrm{C} 5 & -164.0(7) & \mathrm{C} 6-\mathrm{C} 7-\mathrm{C} 8-\mathrm{N} 4 & -55.5(10) \\ \mathrm{N} 2-\mathrm{Cu}-\mathrm{N} 3-\mathrm{C} 5 & 15.1(6) & & 69.7(11) \\ \mathrm{B} 1-\mathrm{Cu}-\mathrm{N} 3-\mathrm{C} 5 & 108.3(6) & & \\ \mathrm{Br} 2-\mathrm{Cu}-\mathrm{N} 3-\mathrm{C} 5 & -72.5(6) & & \\ & & & \end{array}$

Hydrogen-bond geometry $\left(\AA,{ }^{\circ}\right)$

\begin{tabular}{lllll}
\hline$D-\mathrm{H} \cdots A$ & $D-\mathrm{H}$ & $\mathrm{H} \cdots A$ & $D \cdots A$ & $D-\mathrm{H} \cdots A$ \\
\hline $\mathrm{N} 1-\mathrm{H} 1 C \cdots \mathrm{Br} 2^{\mathrm{i}}$ & 0.92 & 2.66 & $3.466(9)$ & 147 \\
$\mathrm{~N} 1-\mathrm{H} 1 D^{\cdots} \cdot \mathrm{Br} 2$ & 0.92 & 2.80 & $3.339(8)$ & 119 \\
$\mathrm{~N} 2-\mathrm{H} 2 C \cdots \mathrm{Br} 1^{\mathrm{ii}}$ & 0.93 & 2.66 & $3.407(8)$ & 138 \\
$\mathrm{~N} 2-\mathrm{H} 2 C \cdots \mathrm{Br} 2$ & 0.93 & 3.01 & $3.519(7)$ & 116 \\
$\mathrm{~N} 3-\mathrm{H} 3 C \cdots \mathrm{Br} 1$ & 0.93 & 2.90 & $3.409(8)$ & 116 \\
$\mathrm{~N} 4-\mathrm{H} 4 C \cdots \mathrm{Br} 2^{\mathrm{i}}$ & 0.92 & 2.60 & $3.515(8)$ & 171 \\
$\mathrm{~N} 4-\mathrm{H} 4 D \cdots \mathrm{Br} 2^{\mathrm{iii}}$ & 0.92 & 2.69 & $3.425(8)$ & 138 \\
$\mathrm{~N} 4-\mathrm{H} 4 D \cdots \mathrm{Br} 1$ & 0.92 & 2.94 & $3.433(8)$ & 115 \\
\hline
\end{tabular}

Symmetry codes: (i) $x+1 / 2,-y+3 / 2,-z$; (ii) $x-1, y, z$; (iii) $x+1, y, z$. 